\title{
Perioperative Nursing Management of an Animal Model of Axial Flow Blood Pump Implantation
}

\author{
Aimei Kang1*, Jinjun $\mathrm{Li}^{2 *}$, Yong Zhou${ }^{1}$, Liu Hu${ }^{1}$, Zhengying Qiu ${ }^{3}$, Xin Zhou ${ }^{1 \#}$ \\ ${ }^{1}$ Wuhan Asian Heart Disease Hospital Affiliated to Wuhan University of Science and Technology, Wuhan, China \\ ${ }^{2}$ Medical College of Wuhan University of Science and Technology, Wuhan, China \\ ${ }^{3}$ Huazhong Agricultural University, Wuhan, China \\ Email: 343128314@qq.com, entry2003@126.com, "zhouxin2007328@163.com
}

Received 19 March 2016; accepted 25 April 2016; published 28 April 2016

Copyright (C) 2016 by authors and Scientific Research Publishing Inc.

This work is licensed under the Creative Commons Attribution International License (CC BY). http://creativecommons.org/licenses/by/4.0/

(c) $\underset{\mathrm{EY}}{\mathrm{B}}$ Open Access

\begin{abstract}
Objective: This study aimed to explore the characteristics of perioperative nursing of experimental goats using self-made axial-flow blood pump implantation and provided theoretical nursing knowledge and practice-based evidence for the clinical application of domestically manufactured artificial cardiac pumps. Methods: Seven experimental goats were used in this study, three for pre-testing and four for the formal experiments. According to the surgical requirements for axial-flow blood pump implantation into the cardiac apex, we creatively designed and made a series of highly practical animal surgical instruments including a composite disassemblable bed for experimental animal transferring and monitoring, a multifunctional animal surgery bed, and portable medical supporting equipment. We also applied for two national invention patents and one utility model patent. Active measures were taken to ensure careful preparation before surgery, close collaboration during surgery, and effective management of complications after surgery. Results: Two of the four experimental goats died during surgery because of a massive hemorrhage caused by distal anastomotic failure and air embolism-induced cardiac arrest caused by air leakage from the outlet into the heart due to poor connection of the auxiliary pressure tap (used to measure left ventricular pressure). The mean survival time of the remaining three experimental goats was 22.7 hours. Conclusion: This study was the first to systematically and comprehensively investigate the perioperative nursing management of axial-flow blood pump implantation using animal models. These findings could greatly promote further clinical applied nursing research of self-made artificial cardiac pump implantation in experimental goats.
\end{abstract}

\footnotetext{
*Joint first author.

\#Corresponding author.
}

How to cite this paper: Kang, A.M., Li, J.J., Zhou, Y., Hu, L., Qiu, Z.Y. and Zhou, X. (2016) Perioperative Nursing Management of an Animal Model of Axial Flow Blood Pump Implantation. International Journal of Clinical Medicine, 7, 270-279. 


\section{Keywords}

\section{Axial-Flow Left Ventricular Assist Devices (LVADs), Goats, Perioperative Nursing Management}

\section{Introduction}

Current clinical evidence shows that heart transplantation is the treatment of choice for severe heart failure. However, the demand for healthy donor hearts greatly outnumbers the supply. According to incomplete statistics, there are approximately 1.5 million patients with end-stage heart failure in China, of which, the majority die while awaiting transplantation due to the shortage of donor hearts. Most of these patients can survive or undergo transplantation surgery if they are provided with suitable circulatory support [1]. In western countries, artificial hearts have been clinically used as bridge-to-transplant devices, which can partially or completely replace defected hearts and restore their biological functions [2]-[10], thus enabling patients to safely pass the waiting period prior to heart transplantation.

Research on ventricular assist devices (VADs) in our country has a late start, and dozens of research institutes have conducted studies on axial-flow VAD in recent years [11] [12]. However, the related studies are still at the animal experiment stage, and various technical difficulties and post-operation complications remain unsolved. In May 2010, our institution began to study and develop implantable axial-flow left ventricular assist devices (LVADs) and eventually completed experiments on seven goats.

Focusing on nursing-based medical care, this study systematically and comprehensively explored perioperative nursing management of self-made axial-flow blood pump implantation in experimental goats. Participation of nursing personnel in this animal study of artificial cardiac pumps allows us to obtain important clinical nursing insights. Therefore, this study can provide theoretical nursing knowledge and practice-based evidence to accelerate the clinical application of domestically manufactured artificial cardiac pumps. Animal Study of Perioperative Care in vivo homemade axial implants artificial heart pump.

\section{Patients and Methods}

\subsection{Experimental Animals and Preparations}

We choose the Boer-Hybrid female goats as animal model for several reasons: the heart volume of goats about $40 \mathrm{~kg}$ was similar with human, which was clear to operate; the Boer-Hybrid was tame to test and collect blood for the researchers. The seven goats were average two years and have a weight between 30 to $48 \mathrm{~kg}$ from sheep farm. Researchers were fixed for establishing emotion with animal, which was beneficial for the nursing [13]. The experimental goats were detected and expelled worms. First of all, preparing 200 or $300 \mathrm{ml}$ goat blood storing in the $4^{\circ} \mathrm{C}-8^{\circ} \mathrm{C}$ refrigerator preoperative. Secondly, detect the electrocardiogram, body temperature, routine blood, routine urine, routine coagulation, D-D2, free hemoglobin, blood sugar and serum biochemistry. Thirdly, preoperative skin preparation and bathing for experimental goats before one day. We shaved the goat body hair when skin preparation for operation and the fixed pipeline. We also make up number on the goats for saving and detecting the experimental materials (Figure 1(a)). Finally, fasting for 24 hours and forbidden drinking for 12 hours before operation. [14] by right lateral given atropine $0.03 \mathrm{mg} / \mathrm{kg}$ and ketamine $3 \mathrm{mg} / \mathrm{kg}$ (intramuscular) before anesthesia. After that, jugular vein catheter was installed and the goats were given the ketamine and propofol (1:2) to maintain the anesthesia. Meanwhile, we also use the homemade laryngoscope to find the trachea opening and inserted the pipe. Finally we pull out the guidewire to inject about $5 \mathrm{ml}$ air to fix the pipe. We connected the anesthesia ventilator to give the isoflurane through the pipe.

\subsection{Experimental Instruments and Equipment}

The apical axial flow device (Figure 1(b)) [15] has the control portion in vitro and implanted portion in vivo. The implanted portion was cylindrical axial flow which was the total volume of $42 \mathrm{ml}$, the weight of $110 \mathrm{~g}$, outside diameter of $26 \mathrm{~mm}$ and a $75 \mathrm{~mm}$ length. We used good compatibility medical stainless steel or titanium as a wall, which was a 18.4 - $20.6 \mathrm{~mm}$ diameter and a $12 \mathrm{~mm}$ diameter grafts guide at the entry and exit ports. The specific parameters are $11000 \mathrm{r} / \mathrm{min}$ speed, $100 \mathrm{mmHg}$ press, $5 \mathrm{~L} / \mathrm{min}$ flow, $12 \mathrm{~W}$ power and $13 \mathrm{~mm}$ diameter. 
(a)

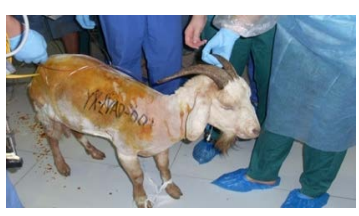

(c)

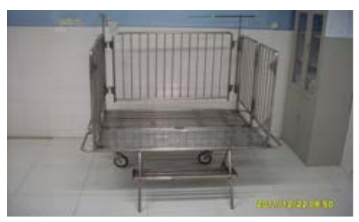

(e)

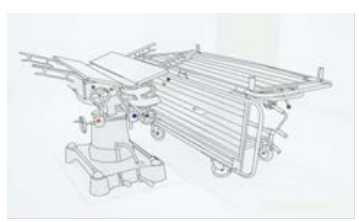

(b)

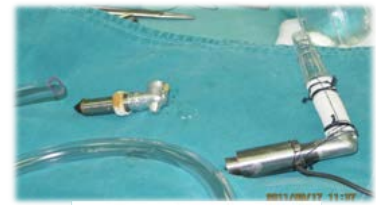

(d)

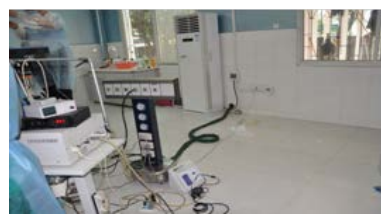

(f)

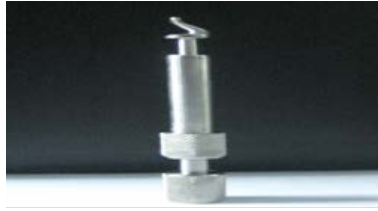

Figure 1. A. Goats; B. The apical axial flow device; C. Removable modular transporter animal care bed; D. Portable medical devices; E. Multiple-functional animal surgery bed; F. Spiral-myocardial punchers.

The vitro control portion was made up of power battery and control circuit which work similar with the common brushless direct current motor. The speed was regulated by input voltage [16].

Experimental surgical instruments were prepared by the goats' weight and height for the deep intro-thoracic operations. The equipment included anesthesia machine (NARKOMED2C12662), multi-parameter anesthetic gas monitors (Vamos), Electrocardiogram (Agilent CM2001, RSM-4101K, MIHON KOHDEN, Japan), and ACT measuring instrument (ACT II, Medtronic Inc, America). There were also several innovative design of equipment such as removable modular transporter animal care bed [17] (National Invention Patent No.ZL 201210042605.8, Figure 1(c)), portable medical devices [14] (National utility model patent No.ZL 201120325472.6 Figure 1(d)), multiple-functional animal surgery bed [18] (National utility model patent No. ZL 201110374405.8, Figure 1(e)) and spiral-myocardial punchers (Figure 1(f)) [19].

We give the implementation of catheterization or bladder puncture, gastrostomy to decompression detection of invasive arterial blood pressure, electrocardiogram, oxygen saturation, and serum biochemical indices. In order to make the activated clotting time (ACT) to $400 \mathrm{~s}$ we give the intravenous heparin $1.5 \mathrm{mg} / \mathrm{kg}$. We also give the Pioneer-5 $1.5 \mathrm{mg}$ and dexamethasone $10 \mathrm{mg}$ preoperatively. After preparation we implemented the left ventricular assist device (LVAD) [20] [21] at the apical and the exit of blood pump was connected with descending aorta by artificial blood vessels. The wire of blood pump was exported from chest incision and placed chest drainage device. By this way we established the left ventricular-LVAD-descending aorta circulation loop (Figure 2(a)): Thoracotomy at the left fourth intercostal space; (Figure 2(b)): The side wall of the descending aorta clamp clip; (Figure 2(c), Figure 2(d)): Descending aorta connected with artificial blood vessels; (Figure 2(e), Figure 2(f): Apical drilling; (Figure 2(g), Figure 2(h)): Implement LVAD at apical and detect the device data.

\subsection{Experimental Drugs}

Hydroxyethyl starch, dexamethasone (HuBei Tian-Yao Pharmaceutical Co., Ltd., China), tranexamic acid (Chongqing Levin US Pharmaceutical Co., Ltd., China), ketamine (Fujian Gu-Tian Pharmaceutical Co., Ltd., China), propofol injection (AstraZeneca Pharmaceutical Co., Ltd., China), Lidocaine (Shandong Hua Lv Pharmaceutical Co., Ltd., China), potassium chloride (Shanghai Hai Hong Pharmaceutical Co., Ltd., China), dicynone (Hubei Tian Yao Pharmaceutical Co., Ltd., China), dopamine (Shanhai He Feng Pharmaceutical Co., Ltd., China), nitroglycerin (Helan Run Hong Pharmaceutical Co., Ltd., China), heparin (Shanghai No. 1 Biochemical Pharmaceutical Co., Ltd., China), protamine (Shanghai No. 1 Biochemical Pharmaceutical Co., Ltd., China), isoflurane (Shanghai Yapei Pharmaceutical Co., Ltd., China). 
(a)

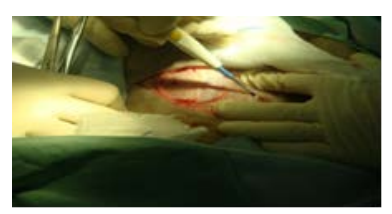

(d)

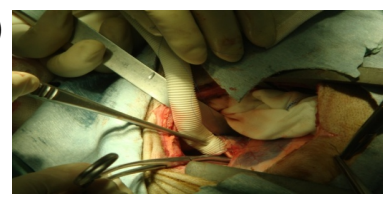

(g)

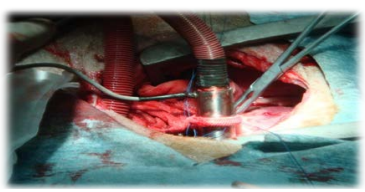

(b)

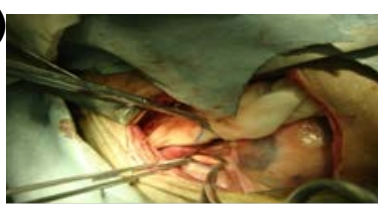

(e)

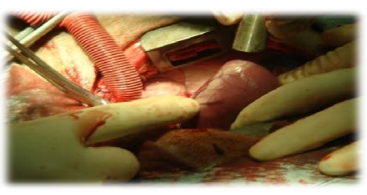

(h)

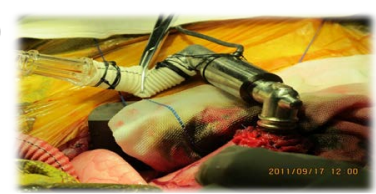

(c)

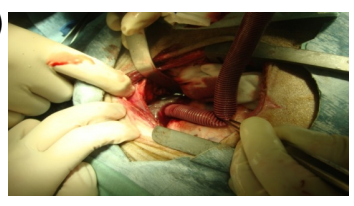

(f)

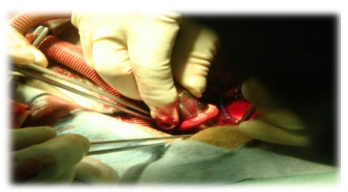

Figure 2. A. Thoracotomy at the left fourth intercostal space; B. The side wall of the descending aorta clamp clip; C, D. Descending aorta connected with artificial blood vessels; E, F. Apical drilling; G, H. Implement LVAD at apical and detect the device data.

\subsection{Method: Perioperative Nursing Management}

\subsubsection{Preoperative Nursing Management}

In order to avoid infection of experimental animals the operation room was cleaned totally and used chlorine disinfectant to wipe flawless and operation table. The operating room was sealed sterilization with ozone sterilizer one day before operation. We take air disinfection with ultraviolet circulation wind. The mops were decontamination for 30min and then air-dry [22].

\subsubsection{Intraoperative Nursing Management}

After general anesthesia we give the goats' conventional skin disinfection and paste electric knife negative plate which was fixed by elastic bandage. In the process of operation we pay close attention to the rate, press, oxygen saturation, breath and salivation. We also reported the parameters for the doctors to regulate the blood pump [23]. When the speed reached to 8000 - $10000 \mathrm{r} / \mathrm{min}$ and the flow reached to 4 - $5 \mathrm{~L} / \mathrm{min}$ the blood pump could maintain the goats' normal pressure (Figures 3(a)-(c)).

\subsubsection{Postoperative Nursing Management}

1) Observe basic vital sign

After anesthesia the body temperature of goats was usually low which easily induced arrhythmia and unstable blood pressure. The temperature of goat named number 1 was 36 Celsius degree. Therefore we sew body-warmers (Figure 3(d)) and used the air conditioning to maintain the goats' body temperature up to 38 - 39.6 Celsius degree. We observed the postoperative vital signs including ruminant, petechial, hematoma and wound [24], and also detected the active clotting time (ACT) regularly, D-D2, free hemoglobin, serum biochemistry and oxygen content for diagnosis to provide the clue.

2) Blood pump management

Because the blood bump was easily dropped we used about 10 centimeter bandage to fix it for two weeks avoiding its pipe anfractuous. Postoperatively we observed the speed, curve and current of blood pump. According to the experimental data the blood pump could maintain the animal for the normal blood pressure when the speed reached to $8000-10000 \mathrm{r} / \mathrm{min}$. When the speed was under $6000-7000 \mathrm{r} / \mathrm{min}$ it cued the thrombus were formed in the pump. At this time we needed to up-regulate the speed to $12000 \mathrm{r} / \mathrm{min}$ about 5 to $10 \mathrm{~min}$ to wash the thrombus.

3) Haemorrhage management

In the four cases of experiments in vivo one of them was dead because of the distal adverse anastomosis. In 


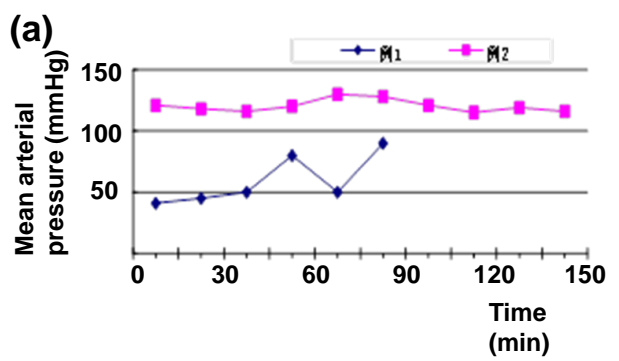

(c)

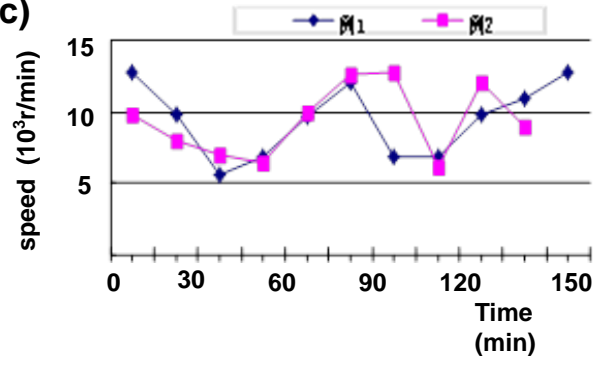

(b)

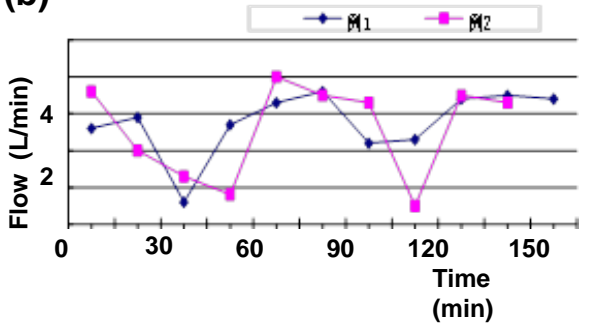

(d)

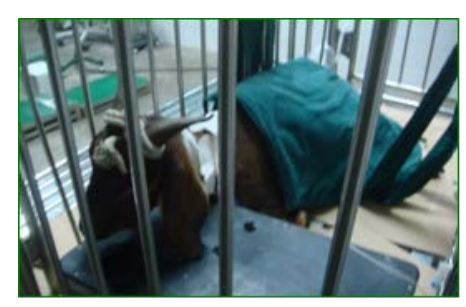

Figure 3. A. 2 cases of experimental goat left heart assist device installed in mean arterial pressure; B. 2 cases of experimental goat left heart assist device installed in the blood pump flow; C. 2 cases of experimental goat left heart assist device installed intraoperatie blood pump rotate speed; D. Body-warmers.

the early postoperative we also detected the activated clotting time (ACT), hemoglobin (Hb) and blood platelet to determine whether given anti-coagulation. Because the goats were monitored on the ground we manufactured a new bed which was easily assembled and transport. In order to avoid hemorrhoea in operation we also developed spiral myocardial perforator (Figure 1(f). By this way there was little blood effusion when operation.

4) Hematolysis management

After operation free hemoglobin (F-HB) and lactate dehydrogenase (LDH) of the second goat were higher than preoperative; after blood pump work we collected urine at several stages and found hematoglobinuria which demonstrated the blood pump may led to mechanical hemolysis (Table 1). The nurse point was to monitor the goats' hematuresis, hematochezia, F-HB and LDH.

5) Heart and kidney management

After operation the first and second goats appeared cough, ruminate, hydrosome and ears droop. Out data (Table 2) show that the urea and creatinine increased in the first and second goat, which demonstrated the goats have the functional damage of heart and kidney.

6) Respiratory system management

The fourth goat was dial out organ intubation and appeared salivate and respiratory arrest. By emergency intubation the breath of goat was recovery, but unfortunately the goat was dead after postoperative several hours. We though the goat with anesthesia after operation should not extubate early. By analyzing our data we concluded the best time for the extubation was ten minutes after anesthesia and spontaneous breathing. We also pay attention to the orthostatic to keep air tube clear and clean the oral nasal secretions.

7) Nutrition support management

After postoperative one hour we only gave water under conscious. Then we feed the goat with fresh leaves to enhance appetite. After postoperative twenty-four hour we feed the goat with green grass which was chopped to improve digestion. According to twenty-four hour intake the goats were also given intravenous rehydration, amino acid and intralipid to supply energy [25].

8) Infection prevention and control maintenance

The infection of goats may be due to air pollution in the operating room or intensive care unit, but also due to pollution from the staff operation or goats' urine. To prevent infection from contamination during invasive procedures in the course of nursing, large doses of antibiotics were early used (cefazolin sodium $1.5 \mathrm{mg}+$ dexamethasone 10mg i.v. before operation, and cefazolin sodium $15 \mathrm{mg} / \mathrm{kg}$ q6h i.v. after operation). Furthermore, adequate drainage of intrathoracic bleeding was noted and dressings were replaced in time for avoiding occurrence of local blood congestion [26]. The Goats blood sugar was positively controlled in preoperative andposto perative, 
Table 1. The plasma free hemoglobin $(\mathrm{mg} / \mathrm{L})$ and lactate dehydrogenase $(\mathrm{u} / \mathrm{L})$ were measured before and after operation in 2 patients with experimental left ventricular assist device.

\begin{tabular}{ccccccccccc}
\hline Goat & Index & Preoperative & \multicolumn{7}{c}{ Postoperative (hour) } \\
\hline No & & & $5 \mathrm{~h}$ & $10 \mathrm{~h}$ & $15 \mathrm{~h}$ & $20 \mathrm{~h}$ & $25 \mathrm{~h}$ & $30 \mathrm{~h}$ & $35 \mathrm{~h}$ \\
\hline 1 & F-HB & 21.6 & 343.4 & 139.5 & 138.8 & 241.3 & 214.6 & 53.6 & 43.8 \\
& LDH & 357 & - & - & - & - & 959 & -- & - \\
2 & F-HB & 36.4 & - & 3142.3 & - & 1969.6 & - & - & - \\
& LDH & 206 & - & - & - & 2042 & - & - & - \\
\hline
\end{tabular}

Table 2. Changes of renal function before and after operation in 2 cases of experimental goats with left ventricular assist device $(\mathrm{mmol} / \mathrm{L})$.

\begin{tabular}{|c|c|c|c|c|}
\hline \multirow{2}{*}{ Goat No } & \multirow{2}{*}{ Index } & \multirow[t]{2}{*}{ Preoperative } & \multirow{2}{*}{$\begin{array}{c}\text { Postoperative } \\
\text { Day } 1\end{array}$} & \multirow{2}{*}{$\begin{array}{c}\text { ostoperative } \\
\text { Day } 2\end{array}$} \\
\hline & & & & \\
\hline \multirow[t]{2}{*}{1} & Urea & 8.15 & 16.92 & 19.76 \\
\hline & Creatinine & 45.00 & 69.00 & 69.00 \\
\hline \multirow[t]{2}{*}{2} & Urea & 8.35 & 16.01 & - \\
\hline & Creatinine & 35 & 173.00 & - \\
\hline
\end{tabular}

notes: Urea normal: $1.87 \sim 8.3 \mathrm{mmol} / \mathrm{L}$, creatinine normal: $45 \sim 84 \mathrm{mmol} / \mathrm{L}$.

and energy materials were supplied in postoperative. A good environment should be maintained by clearing goat droppings and disinfecting air regularly (indoor ventilation twice per day and circulating air ultraviolet disinfection three times per day).

9) Environmental maintenance

a) Temperature and humidity, as two of important factors that influence animals thermal equilibrium, were adjusted respectively at $12^{\circ} \mathrm{C}-24^{\circ} \mathrm{C}$ and $40 \%-70 \%$ according to the physiological requirements of goats. b) Light intensity and photoperiod could affect the physiology and behavior of animals [27]. To avoid glare on animals, ICU installed infrared camera system, using natural light during the day, and using infrared ray at night. c) Goats, as ruminants, could not be good rumination in the case of distraction that affects nutrient absorption and utilization. So the medical staff and workers should keep "four light" [28] (speaking light, operating light, closing light, walking light) during operation. Staffing should be reasonable, and the noisy and sharp metallic sound should avoid when transport the equipment [29].

\subsection{Experimental Data Collecting}

(1) LVAD detector recorded the parameters of blood pump concluding speed, current and curve.

(2) Before operation we detected regular biochenmistry. After operation we regularly detected ACT, D-D2, F-HB, blood sugar, serum biochemistry.

(3) After operation we recorded cycle indicators of goats including temperature, puls, breathing, oxygen saturation, angiosthenia, left ventricular pressure, central venous pressure.

\section{Results}

Death of an experimental goat was defined as its circulatory or pulmonary system ceasing to function for $\geq 6$ minutes. The animal was dissected immediately after its death and the blood pump was removed. The interior as well as opening and exit passages of the blood pump were checked for thrombus formation. Vital organs such as the heart, lungs, liver, kidneys, and spleen were visually inspected for infarction-associated pathological changes and then subjected to pathological sectioning examination. Seven experimental goats were used in this study, three for pre-testing and four for the formal in vivo study. One goat in the formal study died during surgery of a massive hemorrhage caused by a distal anastomotic failure and another goat die for air embolism-induced cardiac arrest caused by poor connection of the auxiliary pressure tap (used to measure left ventricle pressure) that led to air leakage from the outlet into the heart (no post-surgical observation data available). The mean survival 
time of the other three goats was 22.7 hours (experimental goat (4) only survived for 7 hours and no post-surgery observation data were available). The autopsy results showed that the apically inserted assist device was loosely connected to the artificial blood vessel in experimental goat (4). Experimental goat (2) had a broken cardiac catheter that was used to measure left ventricular pressure and died of a postoperative hemorrhage. In experimental goat (1), the blood pump automatically stopped working at postoperative hour 30 . This could be related to thrombus formation within the blood pump, failure of anticoagulant therapy, or wearing of the blood pump-bearing system (Table 3).

\section{Discussion}

\subsection{Issues and Improvement Measures in Nursing Management of Axial-Flow Blood Pump Implantation in Animals}

The sample size of this study was small and the average survival time of the experimental animals was 22.7 hours. However, we managed to summarize the nursing issues encountered during the experimental surgical process and developed specific improvement measures to provide clinical experience for the animal studies of blood pumps in China.

First, one goat died of a hemorrhage during surgery because of distal anastomotic failure. Hence, it was crucial that the surgeon was familiar with the anatomical structures of experimental goats before surgery. During surgery, the surgeon should pay attention to the connection of the distal blood vessels to the pump as well as the auxiliary pressure tap (used to measure left ventricle pressure). The nurses' responsibilities included preparing appropriate surgical instruments according to the experimental goat's chest depth before surgery, being familiar with the surgical procedures, collaborating closely with others during the operation, and monitoring the postoperative drainage volume of the pleural fluid.

Second, our observation of the four goats suggested that red blood cell damage, hemolysis, and thrombosis were the major postoperative complications. Wound bleeding was observed despite the administration of heparin injections and immediate dosage adjustments according to the activated coagulation time, 25-hydroxy-vitamin D-D2, and free hemoglobin levels. Necropsy revealed blood clot formations within the blood pumps and near the connection points between the distal blood vessels and blood pumps. Blood and blood clots had accumulated in the chest cavity. Continuous monitoring showed an increased free hemoglobin level in all animals after surgery and varying levels of hemoglobinuria. These observations could be attributed to bleeding from the wounds and anastomotic sites as well as thrombosis and hemolysis associated with wearing of the axial blood pump-bearing system. Therefore, it was necessary to improve the design of the axial-flow type II blood pump. Moreover, extra attention should be paid to the nursing management of complications such as embolism, especially bleeding monitoring and treatment and anticoagulant therapy.

Third, study data had shown that LVAD implantation complications included infection and multiple organ failure. Thus, emphasis should be put on the management of heart failure complications and monitoring hemodynamic changes during postoperative care. To reach optimal cardiac output, frequent pump pressure adjustments should also be made. Observation and recording of device parameters could provide information about device improvement and development.

Table 3. Causes of death of the four experimental animals.

\begin{tabular}{ccc}
\hline No. & Survival time (h) & Cause of death \\
\hline 1 & 41.7 & $\begin{array}{c}\text { The blood pump stopped working due to thrombus } \\
\text { formation }\end{array}$ \\
3 & 19.5 & $\begin{array}{c}\text { Cardiac catheter inserted into the left ventricle was } \\
\text { broken, leading to a massive postoperative } \\
\text { hemorrhage }\end{array}$ \\
4 & Died during & $\begin{array}{c}\text { Distal anastomotic failure leading to a massive } \\
\text { hemorrhage during surgery }\end{array}$ \\
& 7 & $\begin{array}{c}\text { Loose connection between the assist device and the } \\
\text { artificial blood vessel leading to a massive } \\
\text { postoperative hemorrhage }\end{array}$
\end{tabular}


Fourth, problems encountered during LVAD implantation in experimental goats and the corresponding solutions could guide future studies. During this study, the nursing personnel participated in preoperative discussions to understand the anesthetic and surgical regimens. After surgery, they were also involved in the autopsies of the experimental goats and the discussions of the causes of death with the surgeons and veterinarians. Issues encountered in each experiment were summarized to make improvements and increase work efficiency in the subsequent experiments.

Fifth, all nursing procedures of each experiment should be documented in a timely, subjective, accurate, authentic, complete, and concise manner. For accountability, all record data sheets should be signed and dated by those who wrote or modified them. All experimental records and relevant information of the experimental animals should be compiled and documented accordingly within 72 hours after each experiment for analysis, ensuring the traceability of all data.

\section{2. Animal Ethics}

Animal nursing management affects surgical completion and experiment results accuracy. To improve animal cooperation and achieve a stable success rate, attention to animal welfare is of great significance. Thus, animal care units for experimental goats should be established according to their physiological characteristics. Prior to surgery, nursing personnel should initiate and develop a relationship with animals as early as possible to minimize their unfamiliarity with their surroundings and rejection of humans. This can reduce the possibility of experimental failure related to factors such as noncooperation and tubes slipping off.

\section{3. Effective Management and Cost Control}

This experiment has a high requirement of device number and quality, thus requiring a high investment cost. To effectively reduce the cost, we designed and made our own experimental equipment and instruments that met the needs of the animal cardiac experiment and could be further promoted in other animal and clinical studies. Nursing personnel who will participate in such studies should pay attention to equipment maintenance and minimize possible damage, thus contributing to cost control.

\subsection{Prospective Insight and Hypothesis for Human Application}

Here we summarized our nursing management experience and further obtained some clinical insight on axial-flow blood pump implantation based on seven animal experiments. If clinically proven for use, axial-flow blood pumps are deemed suitable for patients with different types of end-stage cardiac failure. Therefore, hemodynamic changes, such as heart rate, central venous pressure, cardiac index, systolic blood pressure, and pulmonary capillary wedge pressure, should be closely monitored after surgery, while the active administration of vasoactive drugs is necessary to ensure stable circulatory function. Thromboembolism and kidney failure are common complications that require extra attention. Anticoagulant therapy should be initiated early to prevent postoperative red blood cell damage, hemolysis, and thrombosis. All anticoagulants should be given by micro-pumps to ensure precise and even medication delivery, and the dosage should be adjusted accordingly based on the patient's adenosine triphosphate level and bleeding situation. Blood pump parameters should be closely monitored and recorded and then adjusted as needed, and emergency items should be available at all times in case of blood pump dysfunction.

Our country had a late start on vascular assist device research and has yet to report any successful clinical cases. This study is the first to systematically and comprehensively investigate the perioperative nursing management of axial-flow blood pump implantation in animals. Moreover, to meet the surgical requirement of artificial cardiac pump implantation in animals, we creatively designed and made a series of highly practical animal surgical instruments including a composite disassemblable bed for experimental animal transferring and monitoring, a multifunctional animal surgery bed, and portable medical supporting equipment. We have also applied for two national invention patents and one utility model patent. The information collected from this study provides a theoretical and practical foundation for the development and clinical application of domestically manufactured artificial cardiac pumps.

\section{References}

[1] El Banayosy, A., Fey, O., Sarnowski, P., et al. (2001) Midterm Follow up of Patients Discharged from Hospital under 
Left Ventricular Assistance. Journal of Heart and Lung Transplantation, 20, 53-58.

[2] McCarthy, P.M. (1995) Heart Mate Implantable Left Ventricular Assist Device: Bridge to Transplantation and Future Applications. The Annals of Thoracic Surgery, 59, 46-51.

[3] Bank, A.J., Mir, S.H., Nguyen, D.Q., et al. (2000) Effects of Left Ventricular Assist Devices on Outcomes in Patients Undergoing Heart Transplantation. The Annals of Thoracic Surgery, 69, 1369-1374.

[4] Hsu, R.B., Chu, S.H. and Chien, C.Y. (2000) Heart Mate Left Ventricular Assist Device for Long-Term Circulatory Support. Journal of the Formosan Medical Association, 99, 336-340.

[5] Koul, B., Solem, J.O., Steen, S., et al. (1998) Heart Mate Left Ventricu1ar Assist Device as Bridge to Heart Transplantation. The Annals of Thoracic Surgery, 65, 1625-1630.

[6] Rogers, J.G., Butler, J., Lansman, S.L., et al. (2007) Chronic Mechanical Circulatory Support for Inotrope-Dependent Heart Failure Patients Who Are Not Trans-Plant Candidates: Results of the Intrepid Trial. Journal of the American College of Cardiology, 50, 741-747. http://dx.doi.org/10.1016/j.jacc.2007.03.063

[7] Patel, S.M., Throckmorton, A.L., Untaroiu, A., et al. (2005) The Status of Failure and Reliability Testing of Artificial Blood Pumps. ASAIO Journal, 51, 440-451. http://dx.doi.org/10.1097/01.mat.0000169083.90253.3c

[8] Farrar, D.J., Bourque, K., Dague, C.P., et al. (2007) Design Features, Developmental Status, and Experimental Results with the Heartmate III Centrifugal Left Ventricular Assist System with a Magnetically Levitated Rotor. ASAIO Journal, 53, 310-315. http://dx.doi.org/10.1097/MAT.0b013e3180536694

[9] Wieselthaler, G.M.O., Driscoll, G., Jansz, P., et al. (2010) Initial Clinical Experience with a Novel Left Ventricular Assist Device with a Magnetically Levitated Rotor in a Multiinstitutional Trial. Journal of Heart and Lung Transplantation, 29, 1218-1225. http://dx.doi.org/10.1016/j.healun.2010.05.016

[10] Morshuis, M., Schoenbrodt, M., Nojiri, C., et al. (2010) Dura Heart Magnetically Levitated Centrifugal Left Ventricular Assist System for Advanced Heart Failure Patients. Expert Review of Medical Devices, 7, 173-183. http://dx.doi.org/10.1586/erd.09.68

[11] Zhang, Y., Hu, S.S., Zhou, J.Y., et al. (2009) The Study of FW Type of Axial Flow Pump in Vitro Hemolysis and Animal Experiment. Chinese Journal of Clinical Thoracic and Cardiovascular Surgery, 16, 114-117. (In China)

[12] Wu, G.H. (2004) The Axial Heart Assist Device and Its Current Development Situation. Beijing Biomedical Engineering, 23, 306-308. (In China)

[13] Liu, Y.X. and Han, S.F. (2007) The Application Progress of Animal Experiment in Nursing Research. Family Nurse, 5, 1-3. (In China)

[14] Tao, L., Zhou, X. and Zhang, Z.J. (2012) The Manufacture and Application of the Portable Medical Equipment Belt for Clinic. Chinese Medical Equipment Journal, 33, 28-29. (In China)

[15] Li, G.R., Zhu, X.D. and Tian, B.S. (2008) The Study of the Structure and Hydrodynamic Characteristics of the Miniature Apex-Axial Pump. Chinese Medical Equipment Journal, 29, 3-5. (In China)

[16] Zhou, X., Tao, L. and Kang, A.M. (2013) The Analysis of Three Death Cases of Goats in the Left Ventricular Assist Device Experiment. Medical \& Pharmaceutical Journal of Chinese People’s Liberation Army, 25, 46-50. (In China)

[17] Zhou, X., Tao, L. and Zhang, Z.J. (2013) The Manufacture of Animal-Transporting Care Beds with Detachability and Composability. Chinese Medical Equipment Journal, 34, 28-29. (In China)

[18] Zhou, X., Kang, A.M. and Tao, L. (2013) The Manufacture and Application of Multifunctional Animal-Operating Beds. Medical \& Pharmaceutical Journal of Chinese People's Liberation Army, 25, 43-45. (In China)

[19] Tao, L., Zhou, X. and Kang, A.M. (2013) The New Concept of Left Ventricular Assist Device Experiment on Animals. Medical \& Pharmaceutical Journal of Chinese People's Liberation Army, 25, 39-42. (In China)

[20] Qu, Z. (2008) The Modern Mechanical Circulatory Support in the Treatment of Heart Failure. Scientific and Technical Documentation Press, Beijing, 2-7. (In China)

[21] Rao, V., Slater, J.P., Edwards, N.M., Naka, Y. and Oz, M.C. (2011) Surgical Management of Valvular Disease in Patients Requiring Left Ventricular Assist Device Support. The Annals of Thoracic Surgery, 71, 1448-1453. http://dx.doi.org/10.1016/S0003-4975(01)02479-1

[22] Zhai, Q.X., Huang, L.J., Ha, H.X., Jia, L. and Gou, P. (2007) The Maintenance and Management of Animal Clean Operating Room. Laboratory Animal Science, 24, 43-44. (In China)

[23] Kang, A.M., Zhou, X. and Hu, L. (2013) The Nursing and Enlightenment during the Perioperative Period in Laboratory Goats Implanted II Axial Flow Artificial Blood Pump. Journal of Nursing, 20, 41-43. (In China)

[24] Tao, J. and Pei, D.J. (2008) The Nursing of Chronic Heart Failure Patients with the Implantation of Cardiac Resynchronization Therapy Device. Journal of Nursing, 15, 42-43. (In China)

[25] Kang, A.M. and Zhou, X. (2013) Seven Cases of Nursing of Laboratory Goats with the Implantation of Axial Flow Ar- 
tificial Blood Pump. Medical \& Pharmaceutical Journal of Chinese People’s Liberation Army, 8, 55-57. (In China)

[26] Ding, N. and Xu, C.Y. (2006) Five Cases of Intensive Care of Patients with LVAD Device Implantation. Nursing Research, 20, 1266-1267. (In China)

[27] Wang, N., et al. (2010) The Management of Laboratory Animal Based on Animal Benefits. Progress in Veterinary Medicine, 31, 148-152. (In China)

[28] Yang, X. (2010) The Primary Research of the Noise Controlling in Intensive Care Unit of Hospitals. Master's Thesis, Taiyuan University of Science and Engineering, 40. (In China)

[29] Kang, A.M., Zhou, X. and Hu, H. (2013) The Management of the Implantation of LVAD in Goats. Chinese Journal of Comparative Medicine, 23, 42-44. (In China) 